\title{
Understanding the performance of the low energy neutrino factory: the dependence on baseline distance and stored-muon energy
}

\author{
Peter Ballett ${ }^{1, *}$ and Silvia Pascoli ${ }^{1, \text { 田 }}$ \\ ${ }^{1}$ Institute for Particle Physics Phenomenology, Department of Physics, \\ Durham University, Durham, DH1 3LE, United Kingdom.
}

(Dated: September 10, 2018)

\begin{abstract}
We study the physics reach of a Low Energy Neutrino Factory (LENF) and its dependence on the chosen baseline distance, $L$, and stored-muon energy, $E_{\mu}$, in order to ascertain the configuration of the optimal LENF. In particular, we study the performance of the LENF over a range of baseline distances from $1000 \mathrm{~km}$ to $4000 \mathrm{~km}$ and stored-muon energies from $4 \mathrm{GeV}$ to $25 \mathrm{GeV}$, connecting the early studies of the LENF $(1300 \mathrm{~km}, 4.5 \mathrm{GeV})$ to those of the conventional, high-energy neutrino factory design $(4000 \mathrm{~km}$ and $7000 \mathrm{~km}, 25 \mathrm{GeV})$. Three different magnetized detector options are considered: a Totally-Active Scintillator Detector (TASD) and two models of a liquid-argon detector distinguished by optimistic and conservative performance estimates. In order to compare the sensitivity of each set-up, we compute the full $\delta$-dependent discovery contours for the determination of $\theta_{13} \neq 0, \delta_{C P} \notin\{0, \pi\}$ and $\operatorname{sign}\left(\Delta m_{13}^{2}\right)$. For large values of $\theta_{13}$, as recently confirmed by the Daya Bay and RENO experiments, the LENF provides a strong discovery potential over the majority of the $L-E_{\mu}$ parameter space and is a promising candidate for the future generation of long baseline experiments aimed at discovering CP-violation and the mass hierarchy, and at making a precise determination of the oscillation parameters.
\end{abstract}

PACS numbers: $14.60 . \mathrm{Pq}$

\section{INTRODUCTION}

Over the last 50 years, neutrino oscillations have advanced from a tentative hypothesis to a well confirmed physical phenomenon and have been observed in various channels and over a wide range of energy scales. Until recently, the results of several experiments (see, e.g. [1]) had only been able to provide us with measurements of four of the six oscillation parameters: $\theta_{12}, \theta_{23}$, $\Delta m_{21}^{2}$ and $\left|\Delta m_{31}^{2}\right|[2[5]$. This situation has changed over the last few months, with new experimental searches $[6-$ 10] succeeding in measuring the last mixing angle $\theta_{13}$ and confirming that it is large with central values reported by Daya Bay and RENO of $\sin ^{2} 2 \theta_{13}=0.092$ and $\sin ^{2} 2 \theta_{13}=0.113$, respectively [9, 10]. The discovery of non-zero $\theta_{13}$ has increased the likelihood that the resolution of the final uncertainties in the neutrino mixing parameters will be possible within a medium-term experimental program. There are two particularly important unknowns that will dominate this project: measuring the complex phase $\delta$, which governs CP-violating effects in the leptonic sector and is currently unconstrained, and determining the sign of $\Delta m_{31}^{2}$, which dictates the type of neutrino mass-ordering and controls the enhancement of the oscillation probabilities in the Earth due to matter effects for long-baseline and atmospheric neutrinos.

The values of the unknown neutrino mixing parameters may well turn out to have decisive implications for potential extensions of the Standard Model and for understanding the problem of flavor. Improving our knowl-

\footnotetext{
* peter.ballett@durham.ac.uk

$\dagger$ silvia.pascoli@durham.ac.uk
}

edge of these parameters is one of the main aims of the next generation of long-baseline oscillation searches. These experiments are primarily aimed at studying the sub-dominant oscillation probability $P\left(\nu_{e} \rightarrow \nu_{\mu}\right)$, or $P\left(\nu_{\mu} \rightarrow \nu_{e}\right)$. These probabilities have a complex dependence on the oscillation parameters and their extraction from data suffers from the problem of degeneracies [1119]. Various strategies have been devised to weaken or resolve this problem 20 22], for example the energy dependence of the degenerate solutions can be exploited using a wide-band beam [23, 24], matter effects can be used to lift the hierarchy degeneracy [25, 26] whilst, for the measurement of $\theta_{23}$ and its deviations from maximality, the low-energy part of the spectrum is particularly important [27] as well as a precise determination of the disappearance probability.

Amongst future long-baseline neutrino experiments, the Neutrino Factory (NF) [28 30] is a leading design with excellent physics reach. At a NF, muons (or antimuons) produced via pion decay are accelerated to a common energy $E_{\mu}$ (originally around $25 \mathrm{GeV}$ ) and are injected into a storage ring. A significant number of highgamma muons will decay producing a highly collimated beam of neutrinos, whose spectral content is known with high precision. The decay of $\mu^{-}\left(\mu^{+}\right)$in the storage ring produces an initial beam with two neutrino components: $\nu_{\mu}$ and $\bar{\nu}_{e}\left(\bar{\nu}_{\mu}\right.$ and $\left.\nu_{e}\right)$. At the detector, two different signals will be present: for instance with $\mu^{-}$decays, the right-sign muon events which derive from the observation of $\nu_{\mu}$ coming from the disappearance channel and the wrong-sign muon events which are due to $\bar{\nu}_{e} \rightarrow \bar{\nu}_{\mu}$ oscillations. It is necessary to distinguish these two signals in order to reconstruct the appearance probability. This requires a magnetized detector which is able 
to distinguish $\mu^{+}$- and $\mu^{-}$-events. The baseline choice for the conventional NF 31] is a Magnetized Iron Neutrino Detector (MIND) which provides excellent background rejection and very good energy resolution but low detection efficiency for neutrinos with energies in the few $\mathrm{GeV}$ range [32, 33]. Other options include a magnetized Totally-Active Scintillator Detector (TASD) 30] or a detector based on the liquid-argon time projection chamber [34 37]. The latter detectors provide excellent efficiency for neutrinos with energies as low as a few hundred $\mathrm{MeV}$ to $1 \mathrm{GeV}$, excellent energy resolution and low backgrounds but their magnetization is extremely challenging [38].

Before the measurement of $\theta_{13}$, the baseline configuration of the NF [31] used muons with an energy of $25 \mathrm{GeV}$ and two different baselines, at approximately 4000 and $7500 \mathrm{~km}$, with two MIND detectors, a $100 \mathrm{kton}$ one at the shorter baseline and a 50 kton one at the 'magic' baseline [39, 40]. The combination of two distinct baseline distances was designed to help resolve the problem of parameter degeneracies by providing complementary information of the oscillation probability at different points in parameter space. This set-up was optimized for small values of $\theta_{13}$ and has been shown to have an excellent physics reach to $\theta_{13}, \mathrm{CP}$-violation and the mass hierarchy [31, 41, 42].

A set-up, named the Low-Energy Neutrino Factory (LENF), has been proposed as a more conservative option for large values of $\theta_{13}$ 27, 38, 43]. The first proposal for the LENF used a single baseline of $1300 \mathrm{~km}$, corresponding to the Fermilab to DUSEL distance, and consequently, a lower muon energy at around $4.5 \mathrm{GeV}[27,38$, 43]. A detector with good energy resolution and low energy threshold allows the LENF to exploit the rich oscillatory pattern and to achieve an important sensitivity to the oscillation parameters. In the original proposal, the detector of choice was a Totally-Active Scintillator Detector (TASD) magnetized by means of a large magnetic cavern [38]. Other possible detectors include a magnetized liquid-argon time projection chamber, which would be ideal due to its large size and excellent detector performance [34]. The possibility of using a non-magnetized detector has also been studied [44]. In this case, although the event separation between wrong- and rightsign muons is impossible at an individual event level, the use of statistical techniques allows the two channels to be partially distinguished and good sensitivity can be obtained thanks to the high event numbers which are associated with very large non-magnetized detectors. Additional studies are required in order to fully understand the capability of statistical separation of right- and wrong-sign muons and the impact of backgrounds. Studies of the LENF using a Magnetized Iron Neutrino Detector (MIND) have also shown promising performance [42] and could serve as an intermediate step in the development of a future higher-energy facility. However, this set-up is optimized for large energies due to the poor lowenergy efficiency of the MIND detector and consequently the performance typically indicates a generic preference for high energies. A LENF with a TASD has also been considered as part of an incremental neutrino factory program [45]. In this setting, the optimal baseline for a stored-muon energy of $E_{\mu}=4.12 \mathrm{GeV}$ was found to be around $L=1100 \mathrm{~km}$.

The conventional high-energy neutrino factory (HENF) and the LENF are conceptually very similar and are only truly separated by a choice of energy scale. This suggests that the two designs for the neutrino factory should be seen as part of a continuum in energy and distance. Especially in light of the recent measurement of large $\theta_{13}$, which has led to the low-energy design becoming the preferred experimental option, it is important to perform a "green-field" study of the LENF in order to understand the dependence of the sensitivity to the oscillation parameters on the stored-muon energy and the choice of baseline distance and, if possible, to answer the question: what is the optimal LENF?. In this paper, we have performed this study considering ranges for the energy and baseline which interpolate between the HENF and the LENF and providing an understanding of how the NF design transitions between the two regions. In particular, we have conducted a finely-grained scan over the energy and baseline range and have identified new performance indicators for two primary questions: the discovery of $\mathrm{CP}$-violation and of the mass hierarchy. The analysis that we present has, for the most part, been performed without fixing the value of $\theta_{13}$. For the measurements of CP-violation and mass hierarchy this allows us to present results for the full range of possible values of $\theta_{13}$. For completeness, we have also computed the discovery potential for measurements of $\theta_{13}$ itself, as the discussion of this measurement allows the relevant physical effects governing the performance and sensitivities of the neutrino factory to be explored and better understood.

After the completion of our work, a study [46] appeared which also addresses the optimal baseline and stored-muon energy of the LENF. Our work differs from Ref. [46] in a few significant regards. First of all, we have performed a full, simultaneous scan over the $E_{\mu}-L$ parameter space and as such have a diminished reliance on the interpolation of results. Secondly, our study considers three different detector options which helps us to fully understand the potential performance of the LENF. Finally, in this study we have chosen $10^{22}$ total useful muon decays, a range of baseline distances from $1000 \mathrm{~km}$ to $4000 \mathrm{~km}$ and stored-muon energies from $4 \mathrm{GeV}$ to $25 \mathrm{GeV}$. These choices were made to connect previous optimization studies of the LENF $(1300 \mathrm{~km}, 4.5 \mathrm{GeV})$ to those of the HENF (short baseline $\sim 4000 \mathrm{~km}, 25 \mathrm{GeV}$ ). Understanding this parameter space in detail allows the two designs to be viewed as part of a continuum and helps us to unite the previous work on the two designs in a common framework.

This paper is organized as follows. In Section I we discuss the details of our simulation and the assumptions 
we have made in our model of the LENF. In Section III we present the results of our study and provide an analysis of the sensitivities of the LENF across our parameter space. In Section [V] we discuss the performance of the LENF in light of the recently measured value of $\theta_{13}$ and in Section V, we summarize our findings and make a few concluding remarks.

\section{SIMULATION DETAILS}

In this study, we have performed simulations of the physics performance of the LENF over a range of experimental configurations. As our indicators of performance, we have computed the sensitivity of the LENF towards three of the most important potential discoveries for the future generation of neutrino oscillation experiments: the discovery of non-zero $\theta_{13}$, the discovery of CP-violation arising from the Pontecorvo-Maki-Nakagawa-Sakata [47] matrix and the determination of the neutrino mass hierarchy. We have studied the potential for discovery of each of these fundamental phenomena over a range of stored-muon energies given by $4 \leq E_{\mu} \leq 25 \mathrm{GeV}$ and baseline distances by $1000 \leq L \leq 4000 \mathrm{~km}$. This range connects the regions of parameter space traditionally associated with the LENF design 27, 38, 43] to those of the conventional HENF set-up [31].

It has been suggested that $1.4 \times 10^{21}$ muon decays per year is an attainable goal for the lower-energy accelerator facility [48] and this estimate has been incorporated into previous studies of the performance of the LENF [43, 49]. In this work, so as to aid comparison with studies of the standard neutrino factory [41, 42], we have assumed $1.0 \times 10^{21}$ useful muon decays per year per polarity and a run-time of 10 years which is divided evenly between the two polarities ( $10^{22}$ useful muons in total). This value is in accordance with the estimates for the conventional neutrino factory, assuming $10^{7}$ operational seconds per year 31. We have additionally performed simulations assuming the optimized value of $1.4 \times 10^{21}$ muon decays per year per polarity, the data indicate a predictable uniform increase in performance and have been made available online [50]. In Ref. [46], the total number of useful muon decays was taken as $2.5 \times 10^{22}$. This leads to a further increase in statistics and consequently in the discovery potential of the LENF. To understand the performance of the LENF in the context of similar experimental configurations it is important to ensure a fair comparison is made. For this reason, in the analysis that follows we have assumed the conventional $10^{22}$ total useful muon decays.

The detectors in our simulations measure muons arising through both the disappearance $\left(\nu_{\mu} \rightarrow \nu_{\mu}\right.$ or $\bar{\nu}_{\mu} \rightarrow$ $\left.\bar{\nu}_{\mu}\right)$ and the appearance channels $\left(\bar{\nu}_{e} \rightarrow \bar{\nu}_{\mu}\right.$ or $\left.\nu_{e} \rightarrow \nu_{\mu}\right)$. We have chosen not to include the platinum channel (the observation of $\nu_{e}$ and $\bar{\nu}_{e}$ ) as previous work has shown that it offers only marginal improvement of the oscillation parameter sensitivity [43]. The production of $\tau^{+}\left(\tau^{-}\right)$in the detector by the charged-current interactions of incident $\bar{\nu}_{\tau}\left(\nu_{\tau}\right)$ leads to the problem of tau contamination [51, 52]. $\tau$-leptons have a lifetime at rest of $2.9 \times 10^{-13} \mathrm{~s}$ and decay inside the detector into muons with a branching ratio of around 17\% [53]. This effect leads to an increased number of both wrong- and right-sign muons. Due to the form of the oscillation probabilities the number of additional muons in the right-sign channel (e.g. from $\nu_{\mu} \rightarrow \nu_{\tau}$ for $\mu^{-}$in the storage ring) are considerably larger than in the wrong-sign channel (e.g. from $\bar{\nu}_{e} \rightarrow \bar{\nu}_{\tau}$ for $\mu^{-}$in the storage ring). Subsequently, the effect of tau-contamination is most pronounced for measurements which rely on an accurate determination of the disappearance channels, for example in investigations of $\theta_{23}$-maximality [51]. This contamination can lead to serious systematic uncertainties if unaccounted for. However, it has been shown that correctly incorporating this additional source of muons into the analysis of the golden channel, via migration matrices, can resolve the systematic deviations [54]. This has been confirmed in a recent study on the performance of the standard NF [42] where the change in sensitivity produced by correct incorporation of the contamination channel was found to be small. The effect of tau-contamination is also expected to be smaller for lower-energy facilities as the number of neutrinos with energies above the tau production threshold will be reduced. For these reasons, we have omitted the contamination channel in our study.

Our simulations were performed numerically using the GLoBES package [55, 56] which incorporates the Preliminary Reference Earth Model [57, 58] for the computation of the matter density along the baseline. In this package, the oscillation probabilities are computed via numerical diagonalization of the full Hamiltonian assuming three neutrino flavors. However, it is convenient during the analysis of our results to introduce an approximate expression [59] for the golden-channel oscillation probability which is valid up to second-order in $\theta_{13}$, $\alpha \equiv \Delta m_{21}^{2} / \Delta m_{31}^{2}, \Delta m_{21}^{2} L / E$ and $\Delta m_{21}^{2} / E A$

$$
\begin{aligned}
P_{e \mu}= & \sin ^{2} 2 \theta_{13} \sin ^{2} \theta_{23} \sin ^{2}\left(\frac{\Delta m_{31}^{2} L}{4 E}-\frac{A L}{2}\right) \\
+ & {\left[\alpha \sin 2 \theta_{13} \sin 2 \theta_{12} \sin 2 \theta_{23} \frac{\Delta m_{31}^{2}}{2 E A} \sin \left(\frac{A L}{2}\right)\right.} \\
& \left.\quad \times \sin \left(\frac{\Delta m_{31}^{2} L}{4 E}-\frac{A L}{2}\right) \cos \left(\frac{\Delta m_{31}^{2} L}{4 E}+\delta\right)\right] \\
+ & \alpha^{2} \cos ^{2} \theta_{23} \sin ^{2} 2 \theta_{12}\left(\frac{\Delta m_{31}^{2}}{2 E A}\right)^{2} \sin ^{2}\left(\frac{A L}{2}\right) .
\end{aligned}
$$

The first summand in this expression is referred to as the atmospheric term and depends quadratically on $\theta_{13}$. The CP term is second and introduces dependence on $\delta$. The remaining part is called the solar term, which for small values of $\theta_{13}$ can dominate the oscillation probability. As the solar term is independent of $\theta_{13}, \delta$ and $\operatorname{sign}\left(\Delta m_{31}^{2}\right)$, it can lead to a significant loss of sensitivity in the measurement of these parameters if dominant. 
The optimal detector technology for the LENF has not yet been identified and a number of candidate designs remain viable. To facilitate a comparison between the alternative designs, we have performed our simulations for three detectors simultaneously: a Totally-Active Scintillator Detector (TASD) and two different liquid-argon detectors which have optimistic and conservative performance estimates respectively. The TASD concept has been successfully implemented in the MINER $\nu \mathrm{A}$ experiment [60] and a larger scale device has been selected for the upcoming $\mathrm{NO} \nu \mathrm{A}$ 61] design. Our model of the TASD is based upon Ref. [38] and has a fiducial mass of 20 kton, 35 variable-width energy bins and a constant energy resolution of $10 \%$ for both quasi-elastic and non-quasi-elastic events. The efficiency rises linearly from $73 \%$ to $94 \%$ over a range of $0.5 \mathrm{GeV}$ to $1 \mathrm{GeV}$ and then remains constant for higher energies. The background on the golden channel is taken as a constant fraction of $1 \times 10^{-3}$ of the events arising from neutral-current interactions and the same fraction of events from the disappearance channel which accounts for instances of charge misidentification. Both of the liquid-argon detectors are based upon parameters first reported in Ref. [62] and elaborated on in subsequent optimization studies [43] by having a fiducial mass of 100 kton, an energy resolution on quasi-elastic events of $10 \%$ and a flat detection efficiency of $80 \%$. The conservative (optimistic) model has 22 (35) variable-width energy bins and an energy resolution of $20 \%$ (10\%) on non-quasi-elastic events; the backgrounds are taken as a fraction of $5 \times 10^{-3}\left(1 \times 10^{-3}\right)$ of events from both the neutral-current and disappearance channels.

Although a number of large mass-scale liquid-argon detectors have been proposed [37, 63 65], only a few designs [37, 64] discuss extensions to 100 kton. The magnetization of large-volume detectors, of the scale considered in our simulations, is a particular challenge and further research is needed to fully assess the feasibility of the design. Our choice of such large detector volumes (100 kton and 20 kton for liquid-argon and TASD respectively) is designed to provide an optimistic performance estimate which covers the full range of potentialities of the LENF if these technical difficulties can be overcome. It is worth noting that, in our approximation, the performance of the LENF will only depend upon the detector mass through its exposure (number of muon decays $\times$ fiducial detector mass). Using this equivalence, the performance of a 100 kton detector with $1.0 \times 10^{21}$ muon decays per year is expected to be comparable to a 70 kton detector with a larger flux of $1.4 \times 10^{21}$ decays.

We have assumed normal hierarchy to be true throughout our simulations and the known oscillation parameters were chosen to be $\sin ^{2} 2 \theta_{12}=0.3, \theta_{23}=\pi / 4$, $\Delta m_{12}^{2}=8.0 \times 10^{-5} \mathrm{eV}^{2}$ and $\left|\Delta m_{13}^{2}\right|=2.5 \times 10^{-3} \mathrm{eV}^{2}$. The uncertainty on these values was accounted for by allowing the parameters to vary during the minimization procedure: we allowed an uncertainty of $4 \%$ and $10 \%$ for the solar and the atmospheric parameters, respectively. These parameter choices were made in accordance with previous optimization studies of the LENF [43] and are close to the best-fit values and uncertainties from recent global analyses of the existing neutrino oscillation data [2, 4].

\section{RESULTS AND ANALYSIS}

Here we present the performance of the LENF by considering its ability to make three major discoveries. We start by computing the sensitivity to non-zero $\theta_{13}$ which, in spite of recent experimental results, is included to highlight a number of general physical effects which influence the performance of the the neutrino factory. After this, we focus on the discovery of $\delta \in\{0, \pi\}$ and $\Delta m_{31}^{2}>0$. A discussion relevant to the specific case of large $\theta_{13}$ is presented in Section IV.

In this work we compute the discovery potential of nonzero $\theta_{13}$ which is defined as the exclusion of $\theta_{13}=0$ at the $3 \sigma$ confidence level, marginalizing over all other oscillation parameters. For the discovery of CP-violation, we consider the exclusion of all parameter sets with $\delta \in\{0, \pi\}$ (the CP-conserving values) and for the determination of the mass hierarchy, we require the exclusion of all parameter sets with the incorrect hierarchy. To simplify the presentation of our results we use the concept of the discovery fraction, defined as the fraction of true values of $\delta$ at which discovery of the related quantity is possible for a given value of $\theta_{13}$. For each choice of detector, baseline distance and stored-muon energy we have computed the discovery fraction as a function of the true value of $\theta_{13}$ for the three quantities of interest. The full set of discovery plots computed in this study, showing the regions of $\sin ^{2} 2 \theta_{13}-\delta$ parameter space for which discovery is possible, has been made available online [50].

To aid our analysis, we will briefly recall some of the generic factors which influence the variation in performance of the LENF over the $L-E_{\mu}$ parameter space. Maintaining a high flux of neutrinos is essential in longbaseline experiments as this determines the number of events that can be observed at the detector and governs the statistical significance of any observations. The total flux incident on the detector increases with the storedmuon energy as $E_{\mu}^{2}$, whilst decreasing with longer baselines as $L^{-2}$. The observed number of wrong-sign muons is heavily influenced by the oscillation probability, Eq. 1, which introduces an additional dependence on the baseline distance and on the energy of the individual neutrinos, $E_{\nu}$. Observing events which come from the first oscillation maximum is important as it ensures a large signal in the appearance channel. Events from the lowenergy part of the spectrum contain important information on CP-violation, as for these values of $E_{\nu}$ the oscillation probability exhibits a strong dependence on the CP-violating phase $\delta$. Matter effects lead to an enhancement or suppression of the oscillation signal compared to the same process in vacuum. Observing the sign of this difference can provide us with information on the 


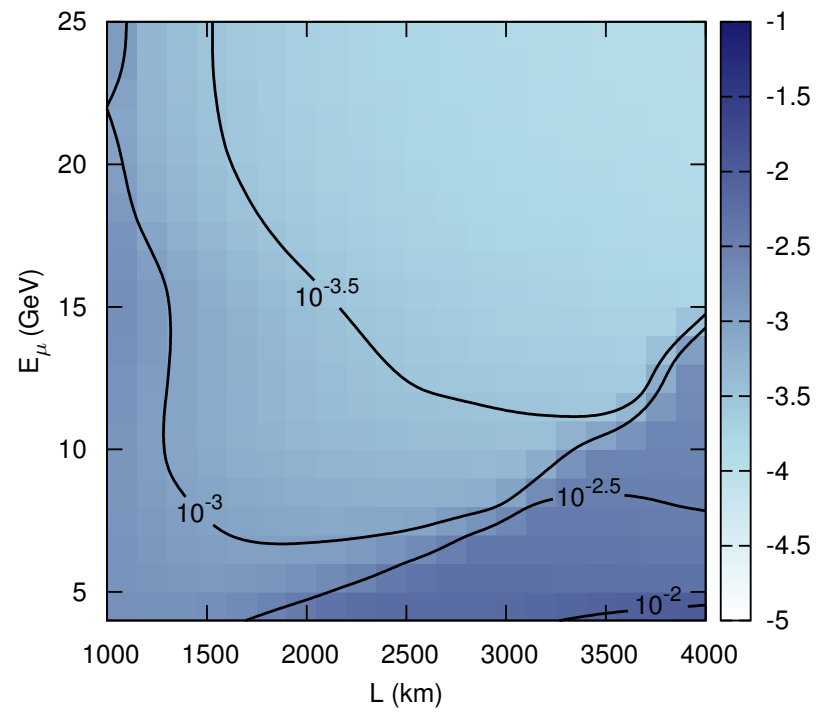

(a) TASD: $100 \%$

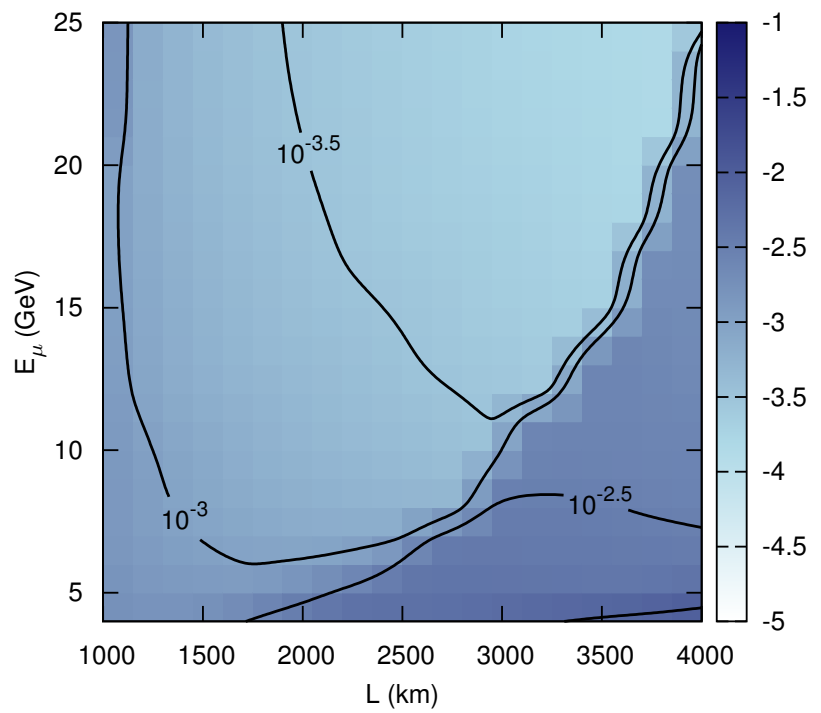

(c) Liquid Ar (conservative): $100 \%$

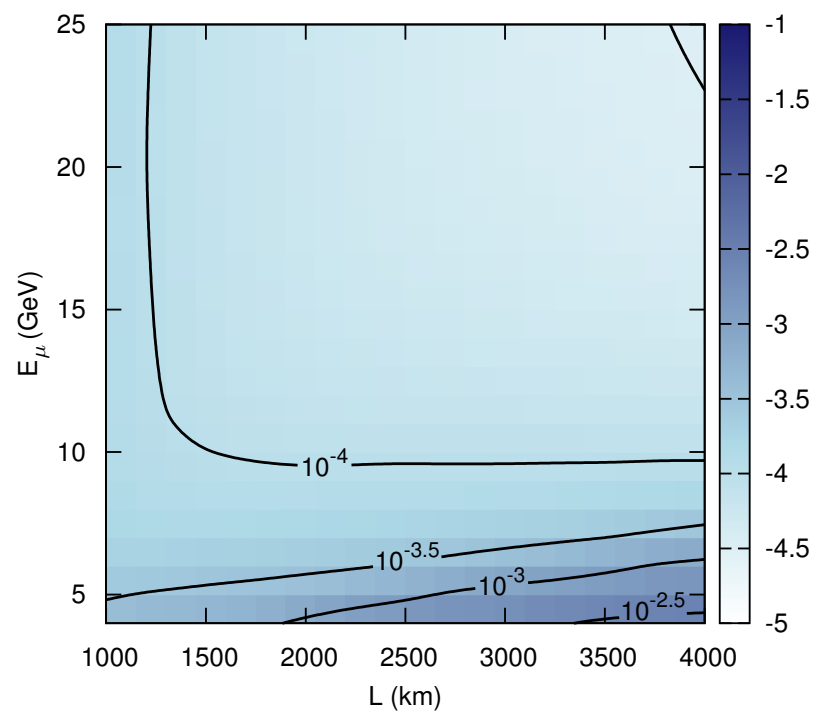

(e) Liquid Ar (optimistic): 100\%

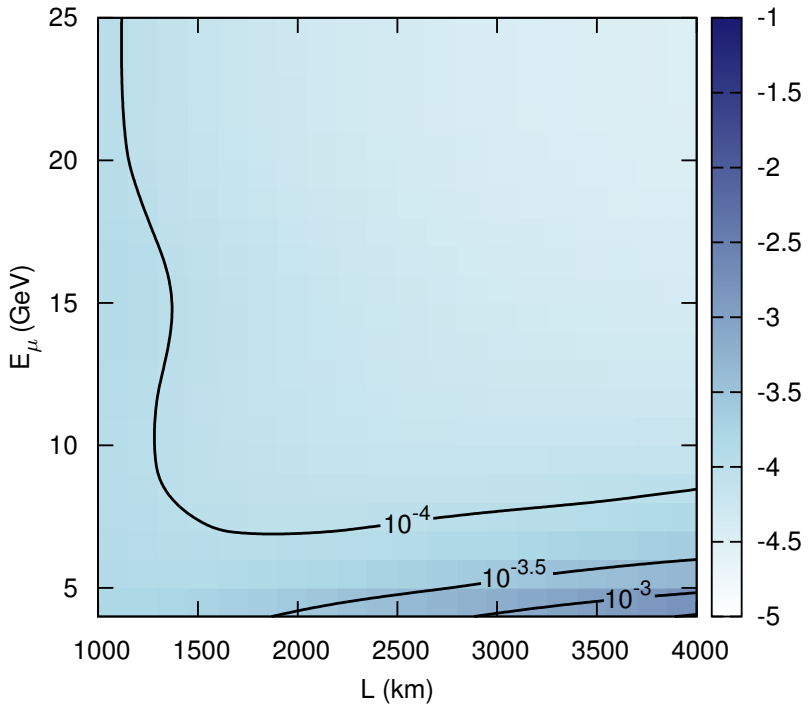

(b) TASD: $0 \%$

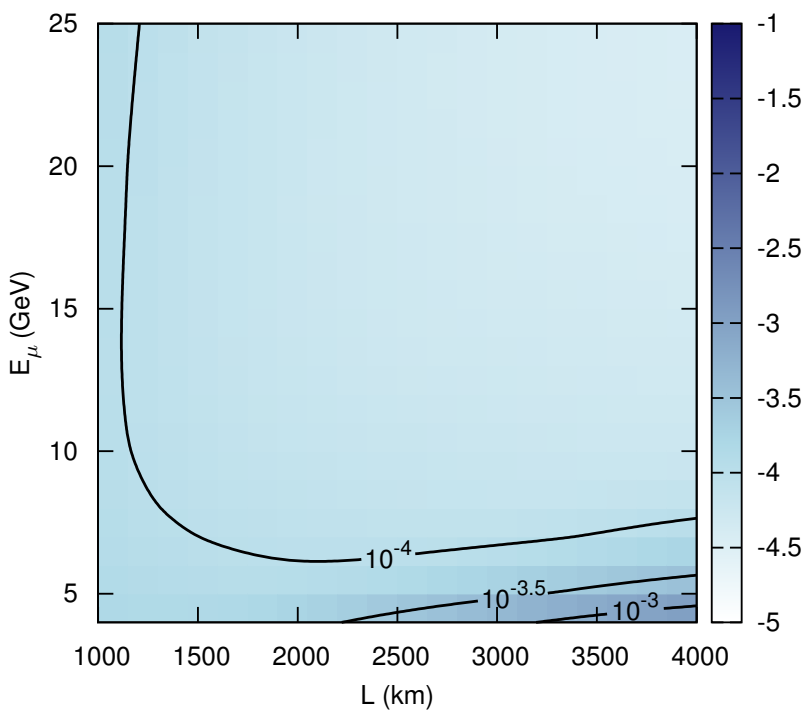

(d) Liquid Ar (conservative): $0 \%$

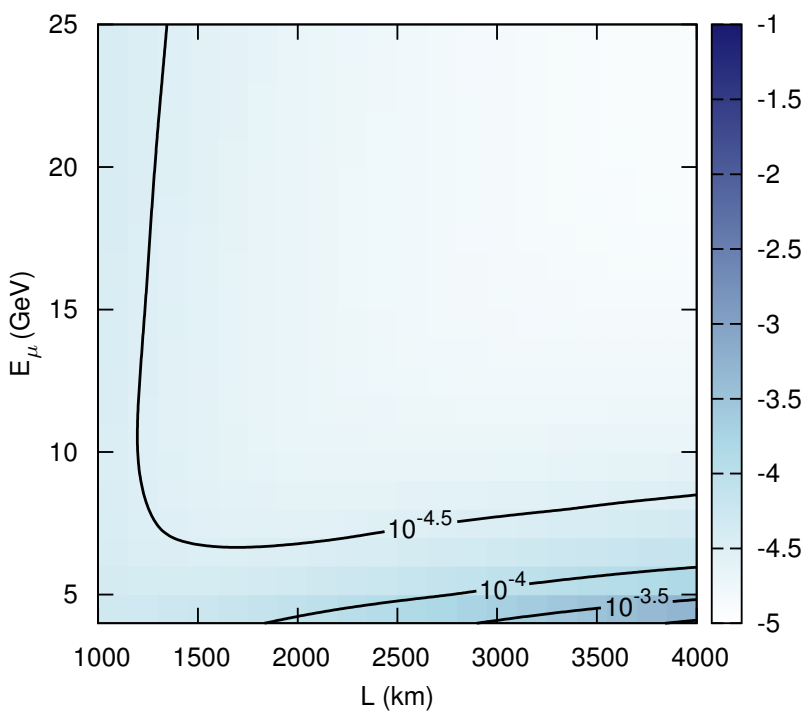

(f) Liquid Ar (optimistic): $0 \%$

FIG. 1. The $\theta_{13}$ discovery range as a function of baseline, $L$, and stored-muon energy, $E_{\mu}$. The left-hand column shows the lowest value of $\sin ^{2} 2 \theta_{13}$ for which the discovery fraction is $100 \%$ for all higher values and the right-hand column shows the lowest value of $\sin ^{2} 2 \theta_{13}$ for which there is a non-zero discovery fraction. The intensity of each point is given by the value of $\log _{10}\left(\sin ^{2} 2 \theta_{13}\right)$. 
neutrino mass hierarchy. In Eq. 1, matter effects are present if $A \neq 0$ and their influence increases with baseline distance and neutrino energy. Sensitivity to a given measurement can also be affected by the presence of degeneracies, the locations of which generally depend on both $L$ and $E_{\nu}$. At the detector, the neutrino-nucleon interaction cross-sections depend on the energies of the incident neutrinos. Flavor-tagged detection is made possible for charged-current interactions by observing the emitted charged lepton. The total cross-section for this channel increases with energy [66, 67] and, assuming that all else is kept equal, leads to an improved number of events at higher-energy facilities.

To understand the variation in performance of the LENF as we vary $L$ and $E_{\mu}$, the individual dependences mentioned above must be considered in combination. In the following sections, we will discuss how these effects can explain the sensitivity of the LENF to non-zero $\theta_{13}$, $\mathrm{CP}$ violation and the mass hierarchy.

\section{A. Discovery of non-zero $\theta_{13}$}

Here we present the results of our simulation which address the ability of the LENF to discover non-zero $\theta_{13}$. In light of the recent measurements of $\theta_{13}$, optimization towards this measurement has become largely peripheral. However, the results in this section, and the accompanying discussion, introduce a number of elements which govern the performance of the LENF in general. As such, it is instructive to first understand this measurement before moving on to the question of CP-violation discovery and the determination of the mass hierarchy. A selection of our results can be seen in Fig. 1 1 where the left column shows, as a function of $L$ and $E_{\mu}$, the lowest value of $\sin ^{2} 2 \theta_{13}$ for which discovery of $\theta_{13} \neq 0$ can be expected, independently of $\delta$, for all higher values. This condition is equivalent to ensuring that all higher values of $\sin ^{2} 2 \theta_{13}$ have discovery fractions of $100 \%$. On the right, Fig. 11 shows the lowest value of $\sin ^{2} 2 \theta_{13}$ which can be discovered for at least one value of $\delta$, which corresponds to a $0 \%$ discovery fraction. These can also be seen as the end points of the $\theta_{13}$ discovery fraction curves seen in previous studies (see for example Fig. 2 in Ref. [42]). Physically, the first of these quantities gives the smallest value of $\theta_{13}$ above which we expect a discovery and provides a conservative estimator of performance. The second quantity is a complementary optimistic estimator and tells us the smallest value of $\theta_{13}$ at which we could possibly make a discovery. The range of these two parameters gives the region of intermediate performance where we find discovery fractions between zero and one; discovery in this region is dependent on the exact value of $\delta$.

In Fig. 1 we see that the TASD expects $\theta_{13}$-discovery to at least $\sin ^{2} 2 \theta_{13} \gtrsim 10^{-3}$ and has the possibility of extending this limit by an order of magnitude. In comparison, the optimistic liquid-argon detector can discover non-zero $\theta_{13}$ down to at least $\sin ^{2} 2 \theta_{13} \gtrsim 10^{-4}$ and possibly as low as $\sin ^{2} 2 \theta_{13} \gtrsim 3 \times 10^{-5}$. The TASD and the conservative liquid-argon detector generally offer comparable sensitivities, which are both worse than those of the optimistic liquid-argon detector. For each detector, we attribute this to different causes. The conservative liquid-argon detector sees a similar total number of events as the optimistic liquid-argon detector however its higher backgrounds lead to a poor signal to background ratio which reduces its sensitivity. In contrast, the TASD has a superior control of the dominant backgrounds to the conservative liquid-argon detector but sees far fewer events due to its smaller size. These two effects reduce the attainable experimental sensitivity by a similar degree.

To further the analysis of our results, we will discuss the four quarters of this parameter space separately. In the short-baseline and low-energy region (SB-LE) with $L \lesssim 2500 \mathrm{~km}$ and $E_{\mu} \lesssim 14 \mathrm{GeV}$, we see $100 \%$ discovery fractions for $10^{-4} \lesssim \sin ^{2} 2 \theta_{13} \lesssim 10^{-2.5}$ depending on the choice of detector technology. In this region, the atmospheric term is relatively suppressed in the $P_{e \mu}$ oscillation probability. This suppression is a result of small $\theta_{13}$ and the relative enhancement of the $\mathrm{CP}$ and solar terms at lower neutrino energies. This leads to a poor performance as the signal becomes decreasingly sensitive to $\theta_{13}$ and the $\mathrm{CP}$ term introduces a more complicated dependence on the oscillation parameters. The decline in performance found towards the very shortest baselines is a consequence of the reduction in oscillation probability at small $L$. This leads to a poor signal-to-background ratio as the number of wrong-sign muons decreases. In the region of short baselines and high energies (SB-HE) with $L \lesssim 2500 \mathrm{~km}$ and $E_{\mu} \gtrsim 14 \mathrm{GeV}$, the larger energies increase the relative importance of the atmospheric term and we see that the situation is marginally improved with respect to the SB-LE region with $100 \%$ discovery fractions for $10^{-4} \lesssim \sin ^{2} 2 \theta_{13} \lesssim 10^{-3}$ depending on detector technology. The overall improvement in this region can be seen as simply a leveling-off of the higher energy improvements of the SB-LE regions: for a fixed baseline distance, there is negligible improvement to be found when moving from $E_{\mu}=15 \mathrm{GeV}$ to $E_{\mu}=25 \mathrm{GeV}$. This plateauing effect is associated with the energy spectrum of the neutrino factory, which rises almost linearly from $E_{\nu}=0$ to its peak at $E_{\nu} \approx 2 E_{\mu} / 3$ and then drops sharply at the kinematic cut-off $E_{\nu}=E_{\mu}$. As we increase the stored-muon energy, the approximately linear tail of this spectrum only decreases slightly and therefore increasing the stored-muon energy at a LENF can be thought of as simply adding higher-energy neutrino events on top of the previous low-energy spectrum. Consequently, the difference in performance between an experiment with a low and a high stored-muon energy can be estimated by determining the importance of the neutrino events occurring with energies between the two stored-muon energies. Moving up through the SB-HE region, the information provided by the additional high-energy neutrinos is decreasingly useful because for higher-energy neutrinos, 
the $L / E$ ratio is smaller and the oscillation probability decreases. This effect leads to a law of diminishing returns, where the discovery reach remains approximately constant. However, as we move to higher energies, the signal to background ratio decreases and with the necessary introduction of additional backgrounds and the slight decrease in the low-energy part of the spectrum, the sensitivity in this region is expected to ultimately be reduced.

For the regions of parameter space with baselines of $L \gtrsim 2500 \mathrm{~km}$, we again divide the parameter space along $E_{\mu}=14 \mathrm{GeV}$ into two quarters: the long-baseline, low-energy region (LB-LE) and the long-baseline, highenergy region (LB-HE). The LB-HE region has in general the best $100 \%$ discovery fractions of all of the parameter space. In this region, the design is approaching the HENF configuration where the signal to background ratio is increased and, thanks to the energy-dependent relative suppression of the solar and $\mathrm{CP}$ terms, the atmospheric contribution to the oscillation probability has a significant influence on the appearance channel. This permits the true value of $\theta_{13}$ to be smaller than in the SB-LE region whilst still providing an appreciable signal and therefore furthering the discovery reach. In contrast, the LB-LE region displays the poorest performance across the parameter space. We find $100 \%$ discovery fractions at around $\sin ^{2} 2 \theta_{13} \approx 10^{-2.5}$ for the TASD and conservative liquid-argon detectors, whilst the optimistic liquid-argon detector has a $100 \%$ discovery fraction for $10^{-3} \lesssim \sin ^{2} 2 \theta_{13} \lesssim 10^{-4}$. The poor performance in this region can be explained as an effect of low statistics: the neutrino flux is reduced as the baseline increases due to the effects of dispersion on the beam. In the LB-LE region this effect is compounded with a small neutrino flux at production. Consequently, the number of wrong-sign muons incident on the detector becomes increasingly restricted for these parameter choices.

In general, the optimal configuration depends upon the magnitude of the true value of $\theta_{13}$. For large values of $\theta_{13}$, as recently confirmed by Daya Bay and RENO 9,10 , all configurations are equally capable of confirming this effect.

The $\theta_{13}$ discovery reaches reported here are consistent with studies performed on the conventional NF [42] and, although making a direct quantitative comparison is difficult, the discovery reaches are of a similar magnitude. This behavior is also reported by Ref. [46] although, due to a difference in exposure, once again the results differ quantitatively.

\section{B. Discovery of CP-violation}

In Fig. 2 we present the CP-violation discovery fraction as a function of baseline and stored-muon energy at different true values of $\sin ^{2} 2 \theta_{13}$ for the TASD and the optimistic liquid-argon detector. The performance of the conservative liquid-argon detector is similar to that of the
TASD and has been omitted from this paper (however, these plots may still be found online [50]). In the SB-LE region, for $\sin ^{2} 2 \theta_{13} \gtrsim 10^{-2}$ we generally see very strong performance with discovery fractions of $70 \%$ to $90 \%$ depending on the choice of detector technology. These sensitivities confirm the expectations behind the original motivation for the LENF: a facility with the ability to measure low-energy neutrino events has access to the oscillation spectrum near the second maximum where CPviolating effects are most pronounced. The simulation has also been performed for smaller values of $\sin ^{2} 2 \theta_{13}$ where the performance of the SB-LE region starts to worsen as the $\delta$-dependent terms in the probability are increasingly suppressed. At the lowest energies, this suppression is compounded by the enhancement of the $\theta_{13^{-}}$ and $\delta$-independent solar term. For $\sin ^{2} 2 \theta_{13} \approx 10^{-4}$ there is only negligible coverage for the TASD however the optimistic liquid-argon detector still maintains a discovery fraction of around $60 \%$ due to its combination of high statistics and strong signal to background ratio. Considering experiments with higher stored-muon energies, we see scant improvement in the SB-HE region as the additional events at high energy provide little information on the parts of the oscillation spectrum which exhibit the most sensitivity to the CP-violating phase. Generally we see discovery fractions of around $70 \%$ to $90 \%$ for $\sin ^{2} 2 \theta_{13} \gtrsim 10^{-2}$, which drops to $60 \%$ to $70 \%$ for $\sin ^{2} 2 \theta_{13} \approx 10^{-3}$. For the smallest values of $\theta_{13}$, the TASD has once again negligible sensitivity whilst the optimistic liquid-argon detector can still determine the effect of CP-violation in $60 \%$ to $70 \%$ of cases.

As in Section IIIA, while the baseline distance is increased there is a decrease in event numbers due to a weakening of the neutrino flux arising from long baselines and this may be compounded by an additional weakening of the flux at low energies. We generally find the lowest discovery fractions in the LB-LE region and this contrast is especially marked in the case of small $\theta_{13}$. However, for long baselines but high energies (LB-HE) we see good sensitivity to CP-violation, especially when $\theta_{13}$ is small. In this region, both the neutrino flux at production and the neutrino-nucleon cross-sections are increased and this helps to mitigate the effect of baseline distance on the event numbers. The additional influence of appreciable matter effects over longer baselines and the inclusion of neutrinos which probe the most CP-sensitive parts of the oscillation spectrum further improve the sensitivity. For $\sin ^{2} 2 \theta_{13} \gtrsim 10^{-3}$, the LB-HE region has comparable discovery fractions to those of the SB-LE region however for $\sin ^{2} 2 \theta_{13} \approx 10^{-4}$ the only significant sensitivity is to be found in the LB-HE region with discovery fractions of $40 \%$ to $70 \%$ for the TASD and optimistic liquid-argon detectors, respectively.

As mentioned previously, the true dependence of our simulations on detector mass, runtime and the number of useful muon decays per year is through their product, referred to as the exposure. In Fig. 3, we can further our understanding of the $\mathrm{CP}$-violation discovery fraction by 

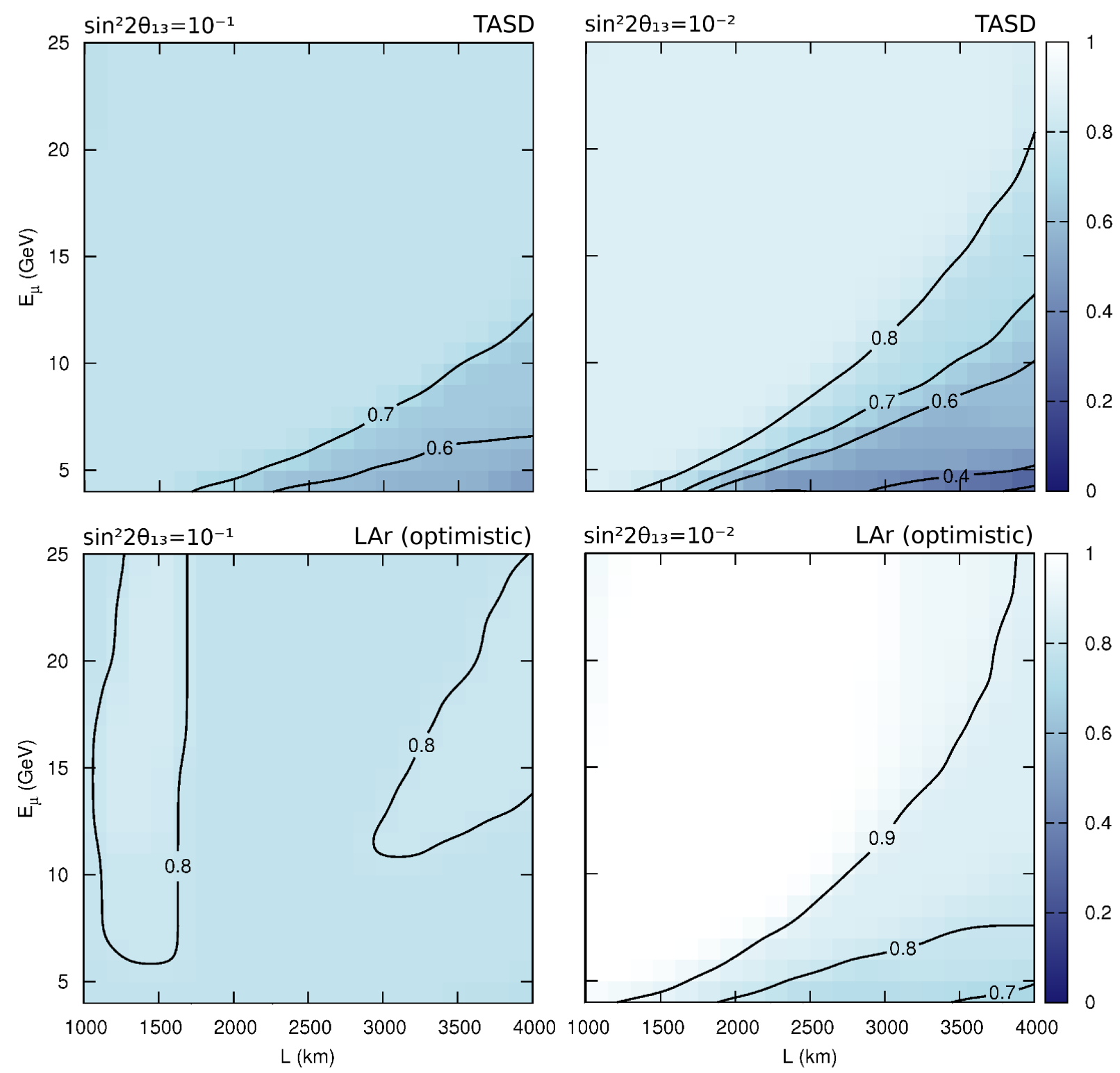

FIG. 2. CP-violation discovery fractions as a function of baseline, $L$, and stored-muon energy, $E_{\mu}$. Each column (row) shows the discovery fraction for a different 'true' value of $\sin ^{2} 2 \theta_{13}$ (detector option) which is given at the top-left (top-right) of each plot.

considering its dependence on this parameter. In general, for large values of $\theta_{13}$ the discovery fraction reaches a plateau for each experimental configuration. This limiting value represents the inherent limitations of the experiment: exposure directly determines the statistics of our experiment and at some point the measurement uncertainties will be dominated by systematic effects for which an increase in statistics can confer only slight improvement to the experiment's sensitivity. The plots in Fig. 3 show the difference in performance for a modest $(10 \%$, left plot) and a more optimistic (1\%, right plot) energy resolution over a range of values of a uniform systematic error on the signal and backgrounds. We see that variations in magnitude of the systematic errors induce the greatest change in the attainable $\mathrm{CP}$-violation discovery fraction. These effects can lead to a significant decline in performance; for $10 \%(1 \%)$ energy resolution, there is a decrease in the discovery fraction of $8 \%(6 \%)$. This limiting influence on the discovery fraction which arises through systematic uncertainties is quite stable to variations under baseline and energy, assuming that these choices do not generate a significant probabilistic suppression of the number of events. This effect helps to explain the observed uniformity in parts of Fig. 2, for example in the SB-HE region. We have also investigated the impact of alternative sources of systematic limitations. For instance, reducing the prior uncertainty associated with the matter density can lead to modest improve- 

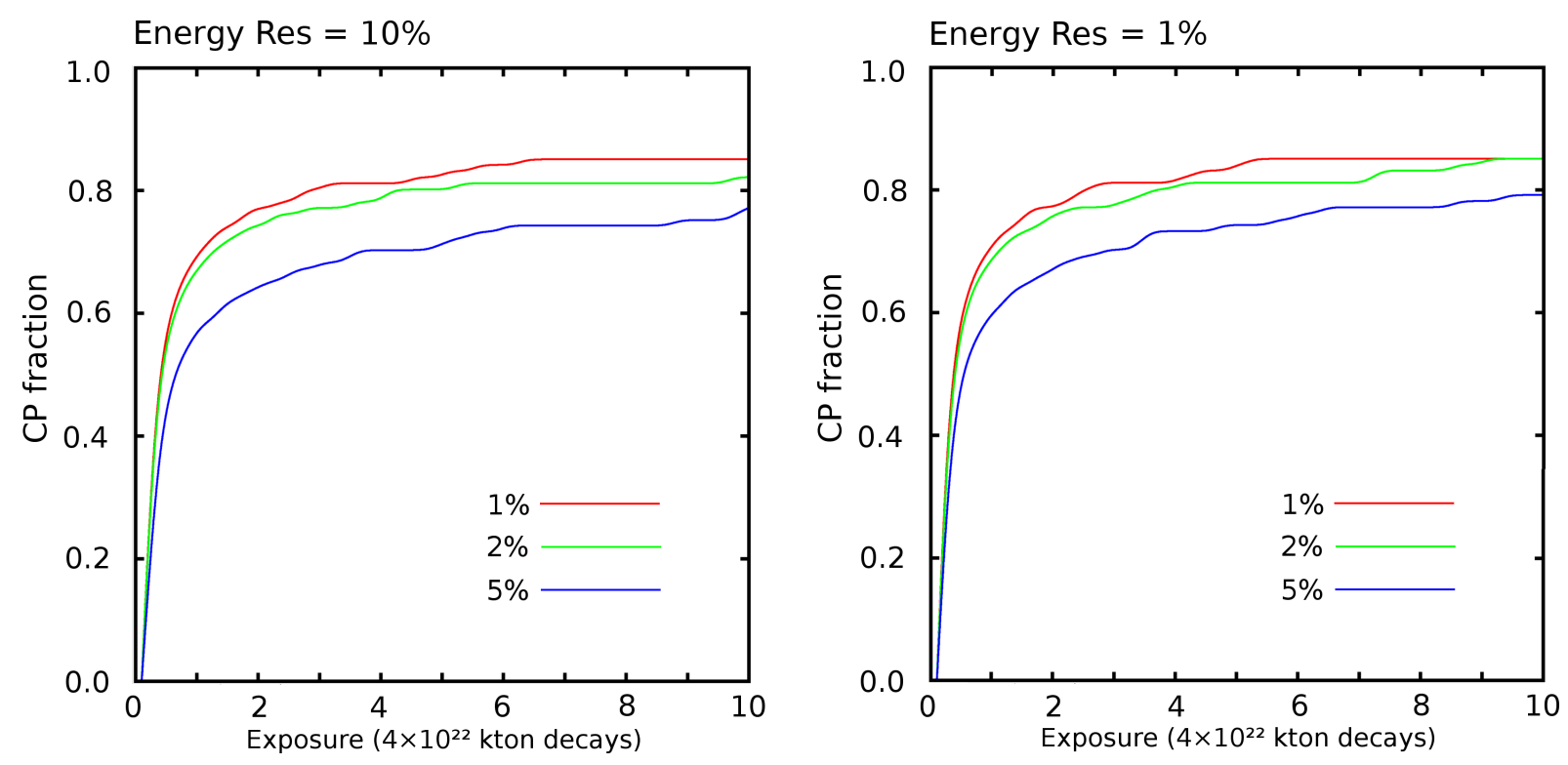

FIG. 3. The CP-violation discovery fraction as a function of exposure for a range of systematic errors. The plot on the left (right) shows the discovery fraction assuming an energy resolution of $10 \%$ (1\%). The scale of the horizontal axis is chosen to coincide with runtime assuming a 20 kton detector and $10^{21}$ useful muon decays per year per polarity. These plots use the TASD and have $\theta_{13}$ set to the Daya Bay best-fit value of $\sin ^{2} 2 \theta_{13}=0.092$ [9]. All otherwise unspecified parameters are as described in Section

ments in sensitivity; however, we find that the unilateral improvement of any one systematic factor leads to little impact on the sensitivity obtained in Fig. 3, it is necessary to reduce all systematics uniformly to significantly further the physics reach. Generally, for the parameter ranges that we have studied, it is the energy resolution and overall systematic error that are responsible for the greatest variation in the attainable CP-discovery fraction.

In common with the discovery of $\theta_{13} \neq 0$, the optimal configuration of the LENF for CP-violation discovery divides into two scenarios depending on the size of $\theta_{13}$. For $\sin ^{2} 2 \theta_{13} \gtrsim 10^{-3}$, provided the LB-LE region is avoided, the CP-violation discovery fractions are almost uniform and the exact configuration is unimportant. As $\theta_{13}$ decreases, the $\delta$-dependent signal is suppressed and appreciable sensitivities can only be found in the LB-HE region where the advantages of the HENF design start to become relevant.

The performance of the single-baseline HENF has been shown [42] to share generic features with our data: the SB-LE region suffers from lower discovery fractions and once $\theta_{13}$ has decreased to around $\sin ^{2} 2 \theta_{13}=10^{-4}$, the only sensitivity can be found in the LB-HE region. The $\mathrm{CP}$-violation discovery fractions of the single-baseline HENF are very similar to those of the LENF with the TASD whereas, for all values of $\sin ^{2} 2 \theta_{13}$ that we have studied, the liquid-argon detector has discovery fractions higher by around 10\%. For a two-baseline HENF, with the longer baseline chosen to be at the magic baseline [42], the comparison changes depending on the size of $\sin ^{2} 2 \theta_{13}$. For $\sin ^{2} 2 \theta_{13} \gtrsim 10^{-2}$ the HENF performs similarly to the LENF with TASD whilst the optimistic liquid-argon detector has higher discovery fractions, once again by around $10 \%$. For values of $\theta_{13}$ in the range $10^{-4} \lesssim \sin ^{2} 2 \theta_{13} \lesssim 10^{-3}$, the HENF starts to outperform the LENF with TASD. However, the performance of the LENF with an optimistic liquid-argon detector remains comparable. Our results in the SB-LE region agree qualitatively with those computed in Ref. 43. for a LENF with $L=1300 \mathrm{~km}$ and $E_{\mu}=4.5 \mathrm{GeV}$ and also agree qualitatively with the recent simulations of the LENF [46].

\section{Determination of the Mass Hierarchy}

In order to present the results of our simulations regarding the determination of the mass hierarchy, we have computed analogous quantities to those used in Section III A In Fig. 4 we have plotted, as a function of $L$ and $E_{\mu}$, the smallest value of $\sin ^{2} 2 \theta_{13}$ at which hierarchy determination is expected to be possible: the $0 \%$ discovery reach. In Fig. 4 we show the smallest value of $\sin ^{2} 2 \theta_{13}$ for which the mass hierarchy can be determined for all higher values of $\sin ^{2} 2 \theta_{13}$, independently of the value of $\delta$ : the $100 \%$ discovery reach. We see that, for most of the parameter space, the TASD provides $100 \%$ discovery reaches of around $\sin ^{2} 2 \theta_{13} \gtrsim 10^{-2}$ and $0 \%$ discovery reaches that are smaller by an order of magnitude. The optimistic liquid-argon detector offers a similar $100 \%$ discovery reach for baselines below $L=2500 \mathrm{~km}$ but, for baselines greater than this, can produce a significantly lower limit between $\sin ^{2} 2 \theta_{13} \gtrsim 10^{-3}$ 

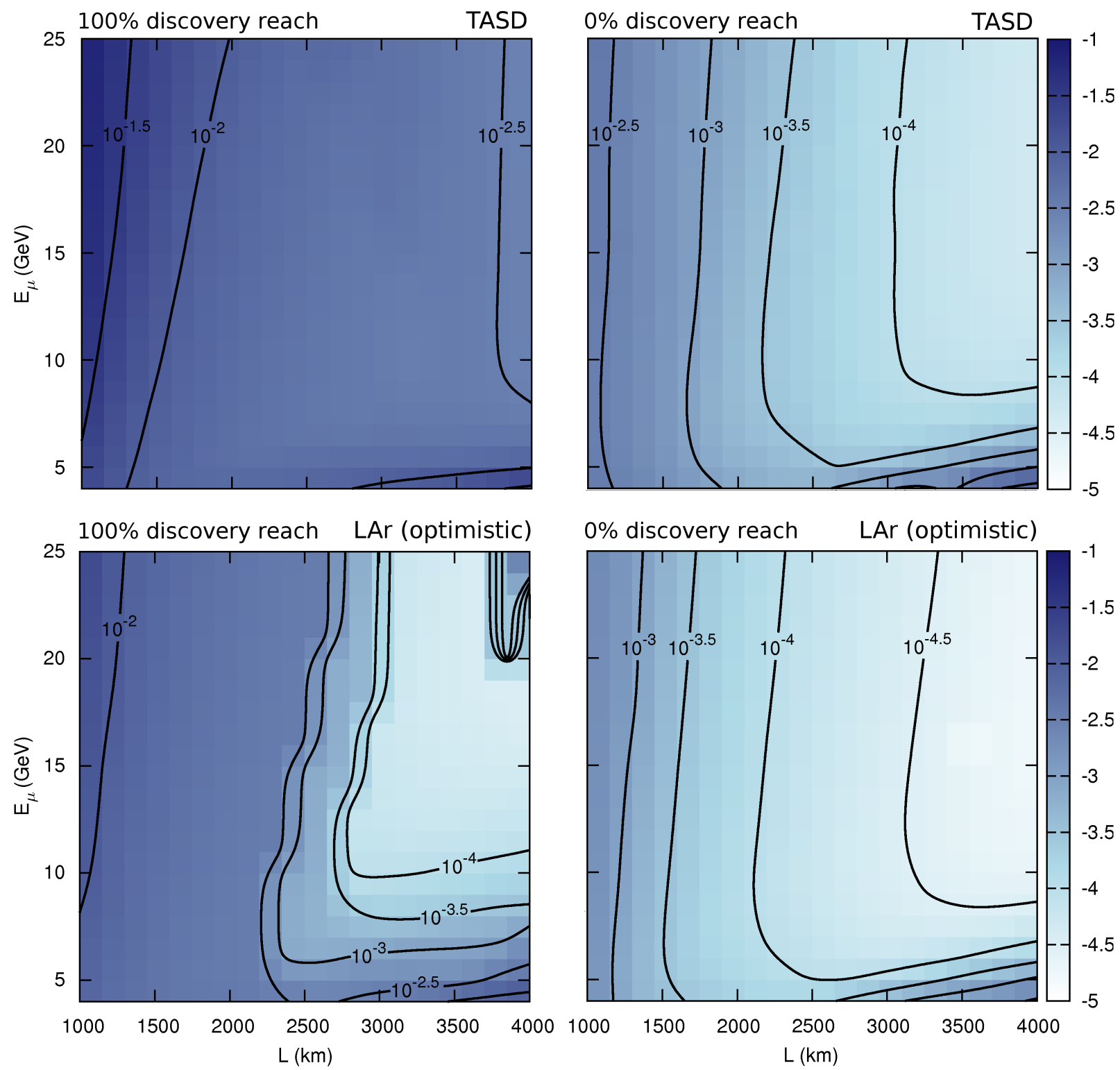

FIG. 4. Hierarchy determination as a function of baseline, $L$, and stored muon energy, $E_{\mu}$, for the TASD (top row) and the liquid-Argon detector with optimistic performance estimates (bottom row). The left column shows the most conservative discovery limit: the lowest value of $\sin ^{2} 2 \theta_{13}$ for which all higher values allow determination of the mass hierarchy independently of $\delta$. The right column shows the optimistic limit: the lowest value of $\sin ^{2} 2 \theta_{13}$ for which there is a non-zero discovery fraction for the exclusion of the incorrect hierarchy. In each plot, the black lines describe regions for which discovery is possible up to the value of $\sin ^{2} 2 \theta_{13}$ as labeled. The intensity at each point is given by $\log _{10}\left(\sin ^{2} 2 \theta_{13}\right)$ of the relevant discovery limit.

and $\sin ^{2} 2 \theta_{13} \gtrsim 10^{-4}$. The $0 \%$ discovery reach in this case is generally $\sin ^{2} 2 \theta_{13} \gtrsim 3.2 \times 10^{-4}$ going down to $\sin ^{2} 2 \theta_{13} \gtrsim 3.2 \times 10^{-5}$ for the longest baselines at around $L=3500 \mathrm{~km}$. It is clear that these plots exhibit a stronger dependence on baseline distance than has been seen in previous plots. This was to be expected as it is well known that matter effects are crucial in lifting the hierarchy degeneracy and that these effects increase with longer baselines. This can be seen in the goldenchannel oscillation probability by considering the difference between the appearance probability for wrong- and right-sign muons. Depending on the neutrino mass hierarchy, one of these channels is suppressed and the other enhanced: this discrepancy grows with longer baselines. An exception to this pattern is found in the LB-LE region where there is a notably poor performance compared to the other regions: the particularly low neutrino flux arising from the combination of long baselines and low stored-muon energies leads to this decreased sensitivity. These results are in qualitative agreement with predictions for the standard NF [42] and with previous studies of the LENF [43, 46]. 
It has been suggested [68] that a low-energy neutrino factory with a "bimagic" baseline of around $L=2540 \mathrm{~km}$ and stored-muon energy of $E_{\mu}=5 \mathrm{GeV}$ would offer a pronounced sensitivity to the neutrino mass hierarchy. This claim was motivated by studies of superbeams [69, 70] which looked for points of $L-E_{\mu}$ parameter space which show a clean separation between the hierarchyconjugate oscillation probabilities. Such a point was found at $L=2540 \mathrm{~km}$ and $E=3.3 \mathrm{GeV}$ where the oscillation probability for the inverted hierarchy is small and independent of $\delta$. In contrast, the oscillation probability for normal hierarchy is much larger and consequently the neutrino fluxes for different mass hierarchies are expected to differ significantly at this point. The same property is also found at $L=2540 \mathrm{~km}$ and $E=1.9 \mathrm{GeV}$ but this time the probability for the normal hierarchy is small and $\delta$-invariant whilst that of the inverted hierarchy is large. It was the existence of two "magic" energies for the same value of $L$ that lead to the idea of a "bimagic" baseline. A neutrino factory with a stored-muon energy of $5 \mathrm{GeV}$ and a baseline of $L=2540 \mathrm{~km}$ would produce a spectrum covering both of these points and this has been shown to lead to a strong sensitivity to the neutrino hierarchy [68]. In Fig. 5 we show our results indicating how the discovery reach depends on baseline distance for a selection of muon energies similar to the bimagic set-up. At a muon storage energy of $E_{\mu}=5 \mathrm{GeV}$ we see evidence for a minimum in the $0 \%$ discovery limit at baseline distances around 2500 to $2800 \mathrm{~km}$. This lower limit corresponds to the optimistic performance of the LENF and it is important to note that there is no corresponding minimum in the conservative estimate shown by the the $100 \%$ discovery limit. We see from the other bands in Fig. 5 how this feature changes as the stored-muon energy increases: the minimum flattens out and drifts to higher baselines. We see that, for all stored-muon energies, a facility with a baseline distance below $L=2500 \mathrm{~km}$ can improve its discovery reach notably by increasing its baseline to at least $2500 \mathrm{~km}$. Beyond this, it appears that if muon storage energies higher than $5 \mathrm{GeV}$ are available then the bimagic choice is not the optimal configuration as the discovery reach can be additionally furthered by increasing both the energy and baseline. Furthermore, it is important to remember that even if stored muon energies are fixed at $5 \mathrm{GeV}$, a baseline of $L \approx 2500 \mathrm{~km}$ only maximizes the optimistic performance of the design and any potential sensitivity advantage would crucially depend on the value of $\delta$.

The behavior in Fig. 5 can be understood in light of our previous analysis. Matter effects are necessary to lift the hierarchy degeneracy and these are increased significantly by the use of long baselines. The reason that this trend does not appear for the $E_{\mu}=5 \mathrm{GeV}$ case is because, at such a low energy, the experiment becomes statistically limited in the LB-LE region and necessitates the move to higher energies. The fact that the configurations near the bimagic baseline do not confer equal improvements for the conservative, $100 \%$ discovery limit is a result of the construction of the bimagic baseline criteria: the degree of contrast between hierarchy-conjugate probabilities is not ensured to be large and its magnitude depends on the exact value of $\delta$. Although for some values the contrast is sizable, for others it is greatly reduced. For example, at the bimagic configuration, when $\delta=0$ the lowest magic energy at $E_{\nu}=1.9 \mathrm{GeV}$ predicts rates which are almost identical for both hierarchies and can offer little discriminatory information. The presence of values of $\delta$ for which the hierarchy distinction is less marked leads to a weaker $100 \%$ discovery limit, which is designed to measure exactly this worst case scenario.

Provided the LB-LE region is avoided, identifying the optimal configuration for measurements of the mass hierarchy reduces to the observation of a simple correlation between the expected magnitude of $\sin ^{2} 2 \theta_{13}$ and the baseline distance. For the smallest values of $\theta_{13}$, configurations in the LB-HE region are necessary. However, for the larger values recently measured by Daya Bay and RENO, the physics reach is quite stable and the exact configuration in our parameter space is unimportant.

\section{DISCUSSION}

In light of the latest experimental results [6 10], the optimization of the LENF for large values of $\theta_{13}$ has become essential. The Daya Bay and RENO experiments can both independently exclude $\theta_{13}=0$ at around $5 \sigma$ and suggest an allowed region of around $0.07 \leq \sin ^{2} 2 \theta_{13} \leq$ 0.14 at $1 \sigma[9,10]$. Our simulations show that these values lie in the optimal region for the performance of the LENF: CP-violation discovery fractions of $80-90 \%$ are attainable in most of the parameter space with an optimistic liquid-argon detector or TASD. This sensitivity can be understood by the low-energy enhancement of the CP term in the golden-channel oscillation probability combined with a relative suppression of the solar term because of the large value of $\theta_{13}$. Additionally, the mass hierarchy will be measurable independently of the choice of $\delta$ for all of the detectors, baselines and stored-muon energies that we have considered in this study. This capability arises because of the effect of the matter potential which generates an enhancement or suppression in the expected number of wrong-sign muons depending on the neutrino hierarchy. For a large value of $\theta_{13}$, a significant number of oscillated events should occur and it is unlikely that the parameter sensitivity will be statistically limited. As the motivation for detectors with very large masses is primarily one of statistics, a large value of $\theta_{13}$ allows a reduction in detector mass whilst maintaining a similar level of performance. Although statistical errors are less relevant with a larger mixing angle, systematic errors become increasingly important. A careful study of the systematic uncertainties will be an important next step in the oscillation analysis.

For a related discussion of the effect of large values of $\theta_{13}$ on the sensitivities of the LENF, see also Ref. [46]. 


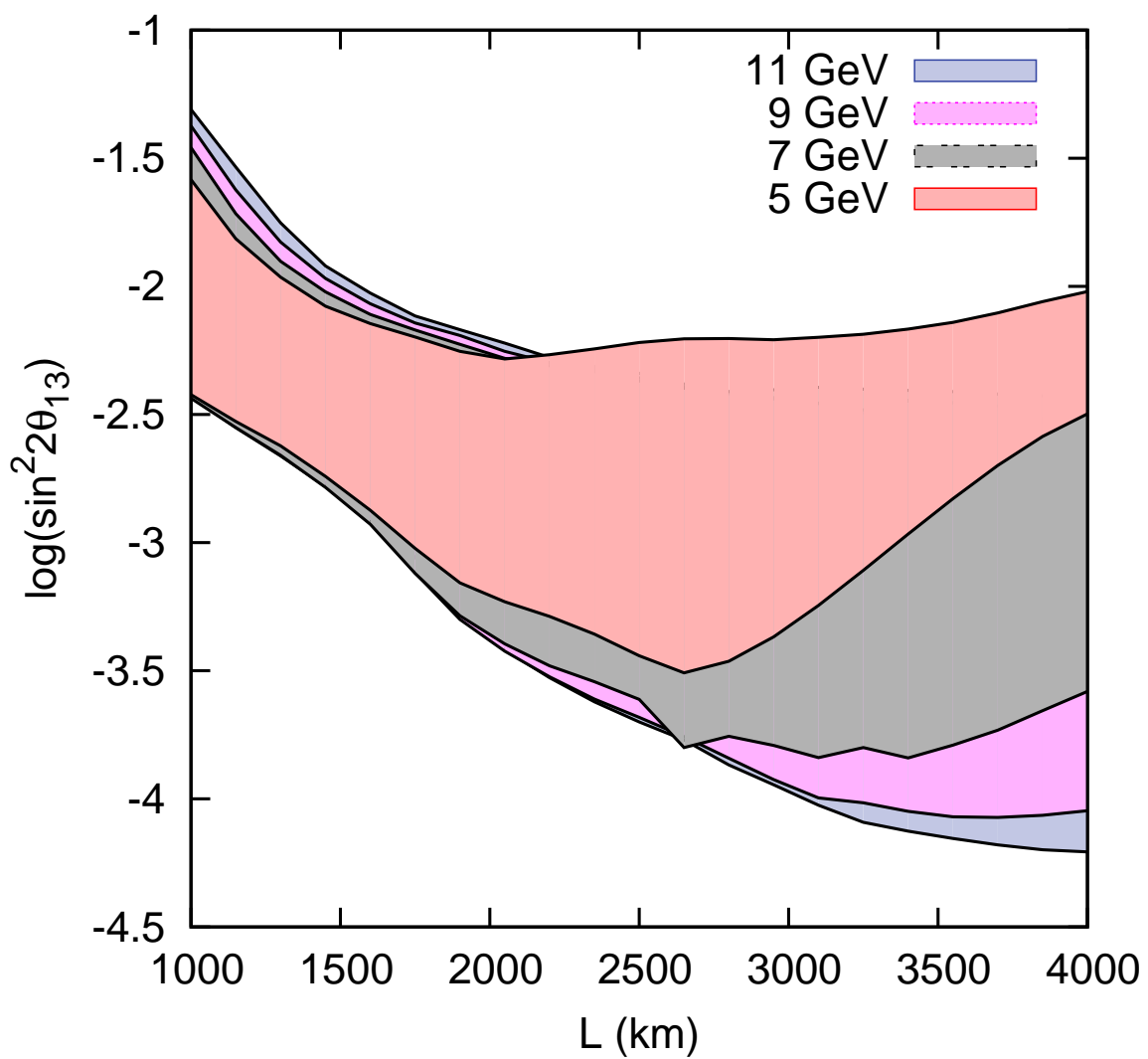

FIG. 5. The hierarchy discovery reach as a function of baseline distance $L$ for the TASD design. For each band the upper (lower) boundary shows the $100 \%(0 \%)$ discovery reach. These correspond to the smallest value of $\log _{10}\left(\sin ^{2} 2 \theta_{13}\right)$ for which the hierarchy can be resolved, independently of $\delta$, for all higher values and the smallest value for which the discovery can be resolved for at least one value of $\delta$, respectively.

\section{CONCLUSIONS}

The Neutrino Factory has previously been studied in two distinct configurations: the conventional HENF uses $25 \mathrm{GeV}$ muons and has two baselines at $4000 \mathrm{~km}$ and $7000 \mathrm{~km}$. More recently, the idea of the LENF has been presented: this uses low-energy muons, typically around 5-10 GeV, and a unique baseline at 1500-2000 km. This set-up exploits the rich oscillatory pattern of the appearance probability and, by mitigating the effect of degeneracies among the unknown neutrino parameters, can provide an excellent physics reach for larger values of $\theta_{13}$. Motivated by recent experimental results, we have performed a "green-field" study of the dependence of the performance of the LENF on the choice of storedmuon energy, $4 \leq E_{\mu} \leq 25 \mathrm{GeV}$, and baseline distance, $1000 \leq L \leq 4000 \mathrm{~km}$, in order to ultimately identify the configuration of the optimal LENF.

In this paper we have presented the results of numerical simulations on the ability of the LENF to answer three questions: is $\theta_{13}$ non-zero, does the neutrino mixing matrix give rise to $\mathrm{CP}$-violating phenomena and what is the correct neutrino mass hierarchy? We find that non-zero $\theta_{13}$ can be discovered for almost all values of baseline and stored-muon energy down to $\sin ^{2} 2 \theta_{13} \gtrsim 10^{-3}$ and we expect limited sensitivity for a further order of magnitude. For CP-violation, discovery fractions of $70 \%$ to $90 \%$ are expected to be attainable for $\sin ^{2} 2 \theta_{13} \gtrsim 10^{-2}$ and of $50 \%$ to $70 \%$ for $\sin ^{2} 2 \theta_{13} \gtrsim 10^{-3}$. We have shown that, provided that the extremal configurations of our $E_{\mu}-L$ parameter space are avoided, specifically very short baselines or long baselines paired with low energies, these performance estimates are quite general and do not require significant fine-tuning of the baseline or the stored-muon energy. The neutrino mass hierarchy is expected to be accessible to the LENF for $\sin ^{2} 2 \theta_{13} \gtrsim 10^{-2}$ with the possibility of discovery generally extending to $\sin ^{2} 2 \theta_{13} \gtrsim 4 \times 10^{-4}$. For the hierarchy determination, the dependence on baseline is clearly seen and if baselines of $L>3000 \mathrm{~km}$ are selected, discovery reach could be extended even further to $\sin ^{2} 2 \theta_{13} \gtrsim 4 \times 10^{-5}$.

We have also considered the potential advantages of the bimagic baseline configuration given by $L=2540 \mathrm{~km}$ and $E_{\mu}=5 \mathrm{GeV}$. We have shown that for this choice of parameters, although a performance maximum is present in the $0 \%$ discovery reach, there is no corresponding maximum in the $100 \%$ discovery reach. This means that, if considered conservatively, such a configuration is of limited benefit for a NF. We have also shown that higher energy configurations can provide an improved discov- 
ery reach and we see a drift in the optimal configuration towards higher energies and baselines.

Now that $\theta_{13}$ has been measured to be relatively large and $\sin ^{2} 2 \theta_{13} \approx 0.09$, we see that the sensitivity of the LENF is comparable for the majority of baseline distance and stored-muon energy arrangements. Furthermore, we expect the results not to be statistically limited, implying that smaller detectors might be considered to provide the required sensitivities and that a detailed study of the impact of systematic errors is required. For these values of $\theta_{13}$, the optimal LENF for the resolution of the most compelling open questions of long-baseline physics has a very broad design. This stability of performance shows that the LENF concept is versatile, with flexibility to accommodate additional design criteria, whilst also providing a strong sensitivity to the most important physical quantities.

\section{ACKNOWLEDGEMENTS}

The authors would like to acknowledge the involvement of Patrick Huber at the commencement of this study and for his valuable comments throughout. This work has been undertaken with partial support from the European Community under the European Commission Framework Programme 7 Design Studies: LAGUNA (Project Number 212343) and EURONU (Project Number 212372). SP acknowledges the support of EuCARD (European Coordination for Accelerator Research and Development), which is co-funded by the European Commission within the Framework Programme 7 Capacities Specific Programme, under Grant Agreement number 227579. PB is further supported by a U.K. Science and Technology Facilities Council (STFC) studentship.
[1] P. Adamson et al. (MINOS Collaboration), Phys. Rev. Lett. 106, 181801 (2011)K. Abe et al. (Super-Kamiokande Collaboration), Phys. Rev. D 83, 052010 (2011)R. Wendell et al. (Kamiokande Collaboration), Phys. Rev. D 81, 092004 (2010)B. Aharmim et al. (SNO Collaboration) arXiv:1109.0763 [nucl-ex]A. V. Derbin (Borexino Collaboration), Phys. Atom. Nucl. 73, 1935 (2010)S. Abe et al. (KamLAND Collaboration), Phys. Rev. Lett. 100, 221803 (2008)

[2] M. C. Gonzalez Garcia, M. Maltoni, and J. Salvado, JHEP 1004, 056 (2010)

[3] G. L. Fogli, E. Lisi, A. Marrone, A. Palazzo, and A. M. Rotunno, Phys. Rev. D 84, 053007 (2011)

[4] T. Schwetz, M. Tórtola, and J. W. F. Valle, New J. Phys. 13, 063004 (2011)

[5] T. Schwetz, M. Tórtola, and J. W. F. Valle arXiv:1108.1376 [hep-ph]

[6] K. Abe et al. (T2K Collaboration), Phys. Rev. Lett. 107, 041801 (2011)

[7] P. Adamson et al. (MINOS Collaboration), Phys. Rev. Lett. 107, 181802 (2011)

[8] H. de Kerret (Double Chooz Collaboration), "The first results from the Double Chooz experiment," Talk presented at LowNu11, Seoul. 9/11/2011

[9] F. An et al. (DAYA-BAY Collaboration)(2012), 5 figures. Version to appear in Phys. Rev. Lett, arXiv:1203.1669 [hep-ex]

[10] J. Ahn et al. (RENO collaboration)(2012), arXiv:1204.0626 [hep-ex]

[11] G. L. Fogli and E. Lisi, Phys.Rev. D54, 3667 (1996)

[12] J. Burguet Castell, M. B. Gavela, J. J. Gómez Cadenas, P. Hernández, and O. Mena, Nucl. Phys. B 608, 301 (2001)

[13] H. Minakata and H. Nunokawa, JHEP 0110, 001 (2001)

[14] V. Barger, D. Marfatia, and K. Whisnant, Phys. Rev. D 65, 073023 (2002)

[15] P. Huber, M. Lindner, and W. Winter, Nucl.Phys. B645, $3(2002)$

[16] H. Minakata, H. Nunokawa, and S. J. Parke, Phys. Rev. D 66, 093012 (2002)
[17] A. Donini, D. Meloni, and S. Rigolin, JHEP 0406, 011 (2004)

[18] M. Aoki, K. Hagiwara, and N. Okamura, Phys.Lett. B606, 371 (2005)

[19] O. Yasuda, New J.Phys. 6, 83 (2004)

[20] T. Kajita, H. Minakata, and H. Nunokawa, Phys. Lett. B 528, 245 (2002)

[21] J. Burguet Castell, M. B. Gavela, J. J. Gómez Cadenas, P. Hernández, and O. Mena, Nucl. Phys. B 646, 301 (2002)

[22] H. Minakata, H. Nunokawa, and S. J. Parke, Phys. Rev. D 68, 013010 (2003)

[23] M. Diwan, D. Beavis, M.-C. Chen, J. Gallardo, S. Kahn, et al., Phys.Rev. D68, 012002 (2003)

[24] P. Huber and J. Kopp, JHEP 1103, 013 (2011)

[25] V. D. Barger, K. Whisnant, S. Pakvasa, and R. Phillips, Phys. Rev. D 22, 2718 (1980)

[26] M. Freund, M. Lindner, S. Petcov, and A. Romanino, Nucl.Phys. B578, 27 (2000)

[27] S. Geer, O. Mena, and S. Pascoli, Phys. Rev. D 75, 093001 (2007)

[28] S. Geer, Phys. Rev. D 57, 6989 (1998)

[29] A. De Rújula, M. B. Gavela, and P. Hernández, Nucl. Phys. B 547, 21 (1999)

[30] A. Bandyopadhyay et al. (ISS Physics Working Group), Rept. Prog. Phys. 72, 106201 (2009)

[31] S. Choubey et al., "International Design Study for the Neutrino Factory, Interim Design Report," (2011), IDSNF-020

[32] A. Cervera, A. Laing, J. Martin-Albo, and F. J. P. Soler, Nucl. Instrum. Meth. A 624, 601 (2010)

[33] A. Laing, Ph.D. thesis, University of Glasgow (2010)

[34] C. Rubbia, "The Liquid Argon Time Projection Chamber: A New Concept for Neutrino Detectors," (1977), CERN-EP-INT-77-08

[35] P. Cennini et al. (ICARUS Collaboration), "ICARUS-II: a second generation proton decay experiment and neutrino observatory at the Gran Sasso Laboratory. Vol. I \& II," (1994), LNGS-94/99

[36] A. Rubbia arXiv:hep-ph/0402110 
[37] A. Rubbia, J. Phys. Conf. Ser. 171, 012020 (2009)

[38] A. D. Bross, M. Ellis, S. Geer, O. Mena, and S. Pascoli, Phys. Rev. D 77, 093012 (2008)

[39] P. Huber and W. Winter, Phys. Rev. D 68, 037301 (2003)

[40] A. Smirnov arXiv:hep-ph/0610198

[41] P. Huber, M. Lindner, M. Rolinec, and W. Winter, Phys. Rev. D 74, 073003 (2006)

[42] S. K. Agarwalla, P. Huber, J. Tang, and W. Winter, JHEP 1101, 120 (2011)

[43] E. Fernández Martínez, T. Li, S. Pascoli, and O. Mena, Phys. Rev. D 81, 073010 (2010)

[44] P. Huber and T. Schwetz, Phys. Lett. B 669, 294 (2008)

[45] J. Tang and W. Winter, Phys.Rev. D81, 033005 (2010), arXiv:0911.5052 [hep-ph]

[46] A. Dighe, S. Goswami, and S. Ray arXiv:1110.3289 [hep-ph]

[47] B. Pontecorvo, Zh. Eksp. Teor. Fiz. 33, 549 (1957)34, 247 (1958)Z. Maki, M. Nakagawa, and S. Sakata, Prog. Theor. Phys. 28, 870 (1962)

[48] C. Ankenbrandt, S. A. Bogacz, A. Bross, S. Geer, C. Johnstone, et al., Phys. Rev. ST Accel. Beams 12, 070101 (2009)

[49] T. Li, Ph.D. thesis, Durham University (2010)

[50] http://www.ippp.dur.ac.uk/ ballett/LENF

[51] D. Indumathi and N. Sinha, Phys. Rev. D 80, 113012 (2009)

[52] R. Dutta, D. Indumathi, and N. Sinha arXiv:1103.5578 [hep-ph]

[53] K. Nakamura et al. (Particle Data Group), J. Phys. G 37, 075021 (2010)
[54] A. Donini, J. J. Gómez Cadenas, and D. Meloni, JHEP 1102, 095 (2011)

[55] P. Huber, M. Lindner, and W. Winter, Comput. Phys. Commun. 167, 195 (2005)

[56] P. Huber, J. Kopp, M. Lindner, M. Rolinec, and W. Winter, Comput. Phys. Commun. 177, 432 (2007)

[57] A. M. Dziewonski and D. L. Anderson, Phys. Earth Planet. Inter. 25, 297 (1981)

[58] F. D. Stacey, Physics of the Earth, 2nd ed. (Wiley, 1977)

[59] A. Cervera, A. Donini, M. B. Gavela, J. J. G. Cadenas, P. Hernández, O. Mena, and S. Rigolin, Nucl. Phys. B $\mathbf{5 7 9}, 17$ (2000)593, 731 (2001)

[60] D. Drakoulakos et al. (Minerva Collaboration) arXiv:hep-ex/0405002

[61] NO $\nu$ A Collaboration, Technical Design Report (2007)

[62] V. Barger, P. Huber, D. Marfatia, and W. Winter, Phys.Rev. D76, 053005 (2007), arXiv:hep-ph/0703029 [hep-ph]

[63] L. Bartoszek et al. arXiv:hep-ex/0408121

[64] D. B. Cline, F. Raffaelli, and F. Sergiampietri, JINST 1, T09001 (2006)

[65] B. Baibussinov, M. Baldo Ceolin, G. Battistoni, P. Benetti, A. Borio, et al., Astropart. Phys. 29, 174 (2008)

[66] P. Lipari, M. Lusignoli, and F. Sartogo, Phys. Rev. Lett. 74, 4384 (1995)

[67] P. Lipari, Nucl. Phys. Proc. Suppl. 112, 274 (2002)

[68] A. Dighe, S. Goswami, and S. Ray, Phys. Rev. Lett. 105, $261802(2010)$

[69] S. K. Raut, R. S. Singh, and S. Uma Sankar, Phys. Lett. B 696, 227 (2011)

[70] A. Joglekar, S. Prakash, S. K. Raut, and S. Uma Sankar, Mod. Phys. Lett. A 26, 2051 (2011) 